1 Square-Root Variable Metric based nullspace shuttle: a 2. characterization of the non-uniqueness in elastic

3

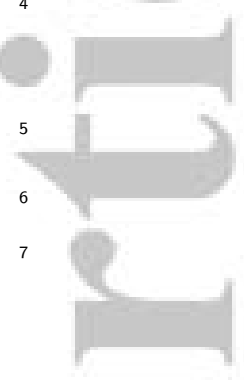

Key Points:

$$
4
$$
\title{
full-waveform inversion
}

\author{
Qiancheng Liu ${ }^{1,2}$, Daniel Peter ${ }^{1}$ \\ ${ }^{1}$ Division of Physical Sciences and Engineering, \\ King Abdullah University of Science and Technology (KAUST) \\ ${ }^{2}$ Department of Geosciences, Princeton University
}

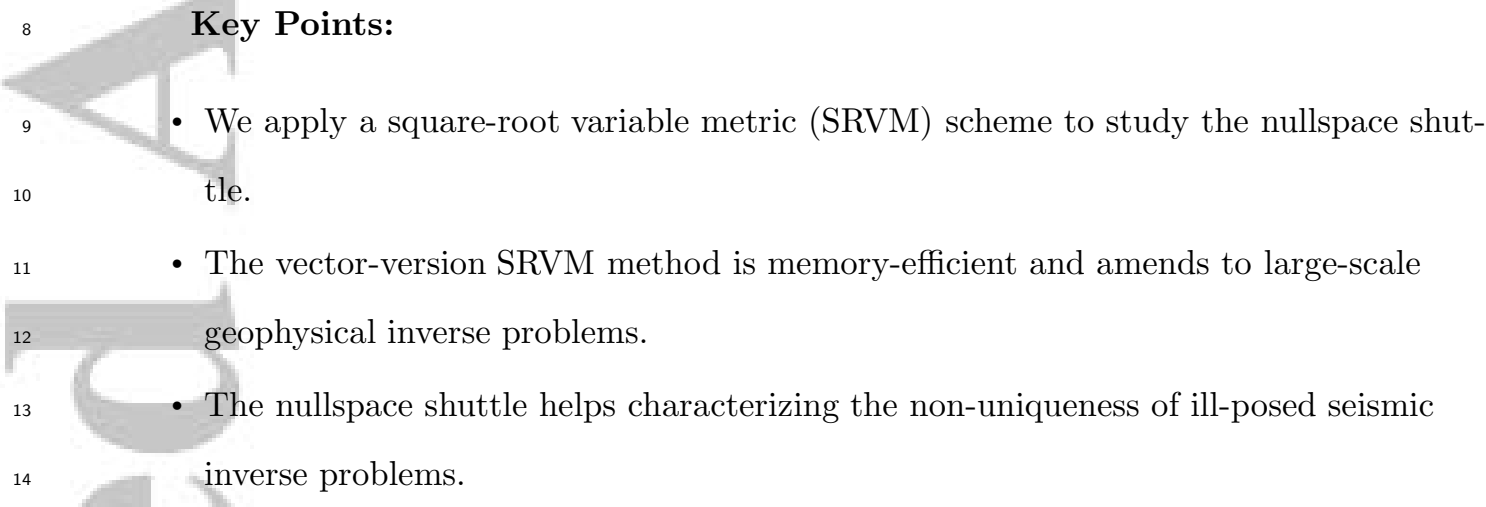

$14 \quad$ inverse problems.

Corresponding author: Daniel Peter, daniel.peter@kaust.edu.sa

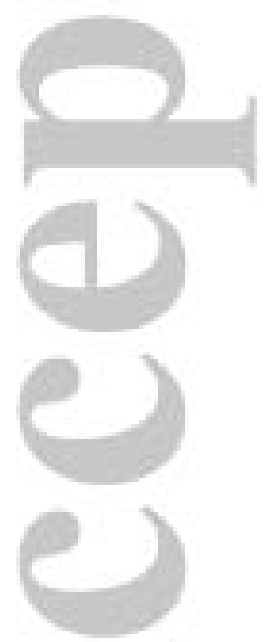

$-1-$

This article has been accepted for publication and undergone full peer review but has not been through the copyediting, typesetting, pagination and proofreading process, which may lead to differences between this version and the Version of Record. Please cite this article as doi: $10.1029 / \quad$ - \% 
Full-waveform inversion (FWI) is for most geophysical applications an ill-posed inverse problem, with non-unique solutions. We examine its non-uniqueness by exploring the nullspace shuttle, which can efficiently generate an ensemble of data-fitting solutions. We construct this shuttle based on a quasi-Newton method, the square-root variable-metric (SRVM) method. The latter provides access to the inverse data-misfit Hessian in FWI for large-scale applications. Combining the SRVM method with a randomised singular value decomposition, we obtain the eigenvector subspaces of the inverse data-misfit Hessian. Its primary eigenvalue and eigenvector are considered to determine the null space of inversion result. Using the SRVM-based nullspace shuttle, we can modify the inverted result a posteriori in a highly efficient manner without corrupting the data misfit. Also, because the SRVM method is embedded through elastic FWI, our method can be extended to multi-parameter problems. We confirm and highlight our approach with the elastic Marmousi example.

\section{Introduction}

29 Full-waveform inversion (FWI) is an important tool in estimating the seismic parameters of the Earth from observed waveform data. As an inverse problem, FWI attempts to seek the "optimal" model which minimizes the data misfit, defined most commonly as the leastsquares residual between observed and synthetic data (Tarantola, 2005). The ever-increasing capabilities in data acquisition and computation enable FWI to constrain seismic models with increasing resolution (Fichtner \& Leeuwen, 2015; Fichtner, Zunino, \& Gebraad, 2018; Virieux \& Operto, 2009). However, due to the limitations of data coverage and the uncertainties in measurements or theories, FWI is an ill-posed problem with non-unique solutions (Fichtner \& Zunino, 2019; Fichtner et al., 2018; Tarantola, 2005; Trampert \& Van Der Hilst, 2005). Indeed, the non-uniqueness of solutions is common to all geophysical inverse problems using actual measurements (Backus \& Gilbert, 1967; Parker, 1977, 1994), with particularly challenging problems such as in seismic, finite-source, magnetic and gravity inversions (Leweke, Michel, \& Telschow, 2018; Mai et al., 2016; Michel \& Fokas, 2008; Snieder \& Trampert, 1999; Vasco, Lane, \& Majer, 1993, see, e.g.,). Thus, further insights can be gained by investigating and quantifying the associated model space.

44 Regarding the non-uniqueness in FWI solutions, the nullspace of the model space provides a collection of models a posteriori within a small misfit tolerance (Deal \& Nolet, 1996; 
nullspace is termed as the "nullspace shuttle", which is guided by the prior information but imposed onto the inverted image a posteriori. The nullspace shuttle exploits the underdetermined nature of the ill-posed problem in seismic inversion. Deal and Nolet (1996) project the model changes onto the space spanned by the nullspace eigenvectors of the data-misfit Hessian. To attack large-scale realistic problems, they obtain the eigenvalues and eigenvectors of the data-misfit Hessian by an iterative method named LSQR (Nolet \& Snieder, 1990; Paige \& Saunders, 1982; Scales, 1987). Hereafter, Rowbotham and Pratt (1997) use the nullspace shuttle to introduce the anisotropy parameter a posteriori to improve the inversion solutions. Deal, Nolet, and van der Hilst (1999) project the difference between the tomographic model and the temperature model onto the nullspace to remove the traveltime violation caused by the temperature components. Trampert and Spetzler (2006) investigate the finite-frequency effects of surface wave tomography in the nullspace. More recently, de Wit, Trampert, and Hilst (2012) generalize the nullspace shuttle to estimate the bounds of the tomographic image to produce a collection of acceptable models. And finally, Fichtner and Zunino (2019) employ a Hamiltonian approach to explore the effective nullspace in nonlinear inverse problems where also the forward modeling equations are nonlinear and the misfit functional non-quadratic.

We attempt here to define the nullspace shuttle in elastic FWI based on the Square-Root Variable Metric (SRVM) method (Hull \& Tapley, 1977; Morf \& Kailath, 1975; Williamson, 1975) together with randomized singular value decomposition (SVD) (Halko, Martinsson, \& Tropp, 2011; Liberty, Woolfe, Martinsson, Rokhlin, \& Tygert, 2007). Tarantola (2005) unburies the fact that SRVM may allow for model resolution analysis and posterior sampling. The original SRVM methods are formulated in a matrix version. Luo (2012) and Liu, Peter, and Tape (2019) rewrite the SRVM method into a vector version to make it memoryaffordable for large-scale FWI problems. Liu and Peter (2019) furthermore incorporate the randomized SVD into the vector-version SRVM approach.

Given a problem which is linear or linearized around the optimum, the model uncertainties can be measured with the resolution (Boschi, 2003) or covariance (Soldati \& Boschi, 2005) matrices. Based on these studies, in this paper, rather than indirectly building the nullspace shuttle with the nullspace eigenvectors of the data-misfit Hessian (Deal \& Nolet, 1996), we directly build the shuttle with the first, primary eigenvector of the inverse data-misfit Hessian. Our research is similar to Meju (2009), who proposes to find a collection of acceptable models with the inverse Hessian by using extremal bound analysis. 


\subsection{Review of the nullspace shuttle method}

The nullspace shuttle method for geophysical tomography was originally proposed by Deal and Nolet (1996), and then got several applications (de Wit et al., 2012; Fichtner \& Zunino, 2019; Rowbotham \& Pratt, 1997). The term nullspace shuttle as introduced by Deal and Nolet (1996) refers to the operator which allows to move from one solution to another without corrupting the data fit. While Fichtner and Zunino (2019) refer to the shuttle "paths", Deal and Nolet (1996) and this paper talk about the shuttle "vehicles". Based on the SVD regarding the data-misfit Hessian or the sensitivity matrix, this approach is used to generate a collection of models with permissible changes but conserve data misfits. We start with the forward problem

$$
\mathbf{d}=\mathbf{f}(\mathbf{m}),
$$

which predicts the observed seismograms $\mathbf{d}$ from a given seismic model $\mathbf{m}$. The linearized forward problem reads

$$
\delta \mathbf{d}=\mathbf{G} \delta \mathbf{m},
$$

with $\delta \mathbf{m}=\mathbf{m}-\mathbf{m}_{0}, \delta \mathbf{d}=\mathbf{d}-\mathbf{d}_{0}$, and $\mathbf{G}=\partial \mathbf{f}(\mathbf{m}) / \partial \mathbf{m}$ being the Fréchet derivative or the sensitivity matrix. The normal equation in the least-squares sense reads

$$
\mathbf{G}^{T} \mathbf{G} \delta \mathbf{m}=\mathbf{G}^{T} \delta \mathbf{d},
$$


where $\mathbf{G}^{T}$ is the transpose of $\mathbf{G}$, and $\mathbf{G}^{T} \mathbf{G}$ is also known as the Gauss-Newton approximation to the data-misfit Hessian (Pratt, 1999; Virieux \& Operto, 2009).

Deal and Nolet (1996) propose to build a set of conservatively filtered models by projecting the model changes onto the generalized nullspace eigenvectors of $\mathbf{G}^{T} \mathbf{G}$. They take an iterative method named LSQR (Paige \& Saunders, 1982) for eigen-decomposition. However, even with the knowledge of the full eigenvectors, we cannot directly define the nullspace from $\mathbf{G}^{T} \mathbf{G}$ due to the following reasons: (i) the definition of the full nullspace is not feasible because it would require many more eigenvectors than those of the effective nullspace (Fichtner \& Zunino, 2019); (ii) in practical applications, data noise may be mapped into the nullspace, making the definition more elusive. Rather than directly deriving from the nullspace eigenvectors, Deal and Nolet (1996) attack this problem from another direction. That is, they first select the eigenvectors that define the range of $\mathbf{G}^{T} \mathbf{G}$, and then subtract the constructed resolution matrix from an ideal resolution matrix, i.e., the identity matrix, to get the nullspace shuttle. That is, given a tolerance $\eta \geq 0$, the perturbations around the "optimal" model $\widetilde{\mathbf{m}}$ may still conserve the data fit as (Fichtner \& Zunino, 2019)

$$
\mathbf{f}\left(\widetilde{\mathbf{m}}+\delta \mathbf{m}_{\text {null }}\right) \leq \mathbf{f}(\widetilde{\mathbf{m}})+\eta
$$

${ }_{96}$ in which $\delta \mathbf{m}_{\text {null }}$ denotes the nullspace shuttle in the model space.

\subsection{Constructing the inverse Hessian via a vector-version SRVM approach}

In Newton's method, the relationship between the model perturbation $\Delta \mathbf{m}$ and the gradient $\mathrm{g}$ is

$$
\Delta \mathbf{m} \approx-\mu \mathbf{H}^{-1} \mathbf{g}^{T}=-\mu \mathbf{B} \mathbf{g}^{T}
$$

where $\mu$ is the one-dimensional search step, $\mathbf{H}$ the Hessian, and $\mathbf{B}$ the inverse Hessian. The standard Davidon-Fletcher-Powell (DFP) method (Fletcher \& Powell, 1963) gives an iterative approximation to the inverse Hessian

$$
\mathbf{B}_{k+1}=\mathbf{B}_{k}-\frac{\mathbf{B}_{k} \Delta \mathbf{g}_{k} \Delta \mathbf{g}_{k}^{T} \mathbf{B}_{k}}{\Delta \mathbf{g}_{k}^{T} \mathbf{B}_{k} \Delta \mathbf{g}_{k}}+\frac{\Delta \mathbf{m}_{k} \Delta \mathbf{m}_{k}^{T}}{\Delta \mathbf{g}_{k}^{T} \Delta \mathbf{m}_{k}}
$$

$$
\text { with } \Delta \mathbf{g}_{k}=\mathbf{g}_{k+1}-\mathbf{g}_{k} \text { and } \Delta \mathbf{m}_{k}=\mathbf{m}_{k+1}-\mathbf{m}_{k} \text {. A workflow of a vector-version SRVM }
$$

algorithm (Liu et al., 2019) is expressed as 


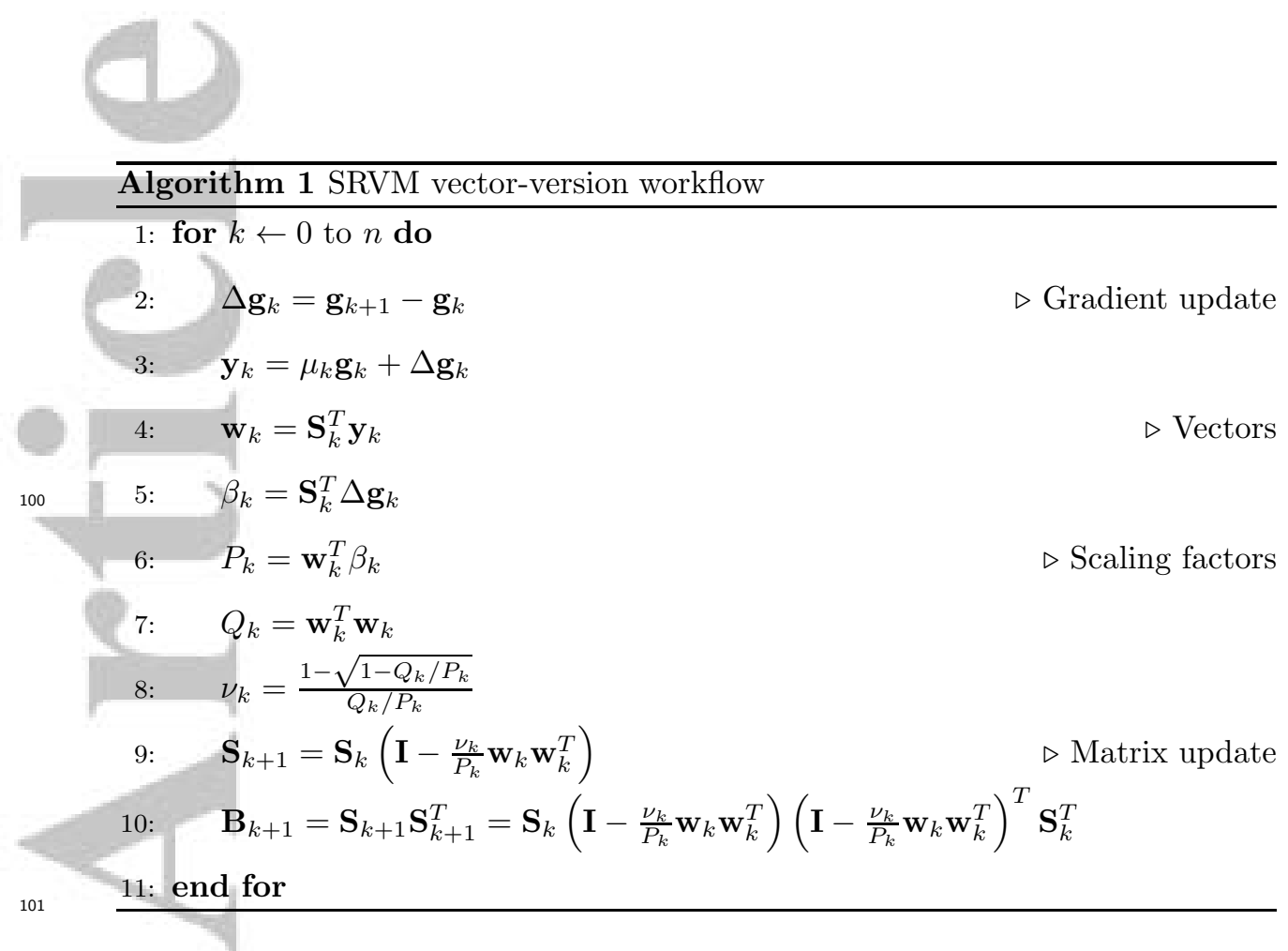

In the vector-version SRVM algorithm, we only need to keep one vector $\mathbf{w}_{k}$ and one scalar $\frac{v_{k}}{P_{k}}$ additionally per iteration, which is memory-efficient even for large-scale problems. The initial guess of $\mathbf{S}_{0}$ is an identity matrix $\mathbf{I}$ such that all the operations are expressed in vectors except for those regarding $\mathbf{S}_{k+1}$ and $\mathbf{B}_{k+1}$. Note that in the SRVM-based elastic FWI, we determine the search direction with the gradient via a matrix-vector product operation $\mathbf{S}_{k+1} \mathbf{S}_{k+1}^{T} \mathbf{g}_{k+1}$, which is finally in a vector version (Liu et al., 2019). Once the SRVM-based FWI converges after $n$ iterations, we have $\mathbf{B}_{n+1}$, a positive-definite approximation to retrieve the inverse Hessian over all the iterations (Liu \& Peter, 2019), as follows

$$
\mathbf{B}_{n+1}=\mathbf{B}_{0}+\sum_{k=0}^{n}\left(\frac{\Delta \mathbf{m}_{k} \Delta \mathbf{m}_{k}^{T}}{\Delta \mathbf{g}_{k}^{T} \Delta \mathbf{m}_{k}}-\frac{\mathbf{B}_{k} \Delta \mathbf{g}_{k} \Delta \mathbf{g}_{k}^{T} \mathbf{B}_{k}}{\Delta \mathbf{g}_{k}^{T} \mathbf{B}_{k} \Delta \mathbf{g}_{k}}\right),
$$

in which $\mathbf{B}_{0}$ is usually chosen as an identity matrix $\mathbf{I}$, an initial guess of the inverse Hessian. Also, $\mathbf{B}_{0}=\mathbf{I}$ acts as a stabilizer to ensure a sufficiently stable $\mathbf{B}_{k}$ during iterative inversion (Nocedal \& Wright, 2006). As the iterations proceed, $\mathbf{B}_{k+1}$ tends to the inverse Hessian (Tarantola, 2005). Equation (7) indicates that the history information about the gradients and the model updates only contributes to $\mathbf{B}_{n+1}-\mathbf{B}_{0}$, so we retrieve the approximated inverse Hessian by (Liu \& Peter, 2019)

$$
\mathbf{H}^{-1}=\mathbf{B}_{n+1}-\mathbf{I}
$$




\section{(1)}

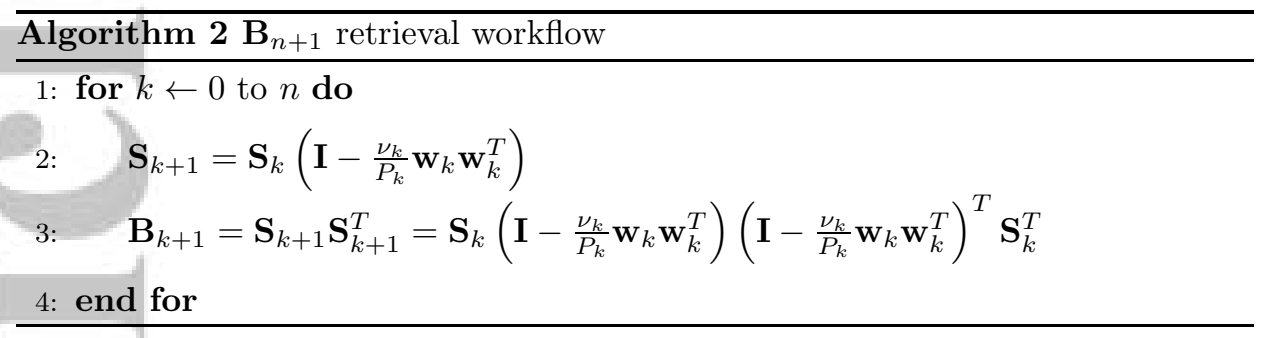

${ }_{106}$ The outer product $\mathbf{w}_{k} \mathbf{w}_{k}^{T}$ in algorithm (2) spans a matrix, which is not easy to store 107 and operate for large-scale problems. In the next subsection, we will seek solutions from randomised SVD to facilitate the information retrieval from algorithm (2).

\subsection{Randomised SVD into SRVM approach}

The SRVM method is a low-rank approach to reconstruct $\mathbf{H}^{-1} \approx \mathbf{B}_{n+1}-\mathbf{I}$. To further facilitate the sampling from $\mathbf{H}^{-1}$, we employ the randomised SVD (Halko et al., 2011; Liberty et al., 2007) to efficiently factorize $\mathbf{H}^{-1}$ into eigenvalues and eigenvectors. Given a symmetric matrix $\mathbf{Z}$ of dimension $M \times M$ and a set of random vectors $\mathbf{X}$ of dimension ${ }_{114} \quad M \times N_{R}$, where $N_{R}$ is the estimated rank order of $\mathbf{Z}$, the randomised SVD runs in a workflow 115 described below

\section{Algorithm 3 Single-pass randomised SVD algorithm}

1: $\mathbf{Y}=\mathbf{Z X}$

2: $\mathbf{Q R}=\mathbf{Y}$

116

$$
\text { 3: } \mathbf{A}\left(\mathbf{Q}^{T} \mathbf{X}\right)=\mathbf{Q}^{T} \mathbf{Y}
$$$$
\text { 4: } \mathbf{U} \Lambda \mathbf{U}^{T}=\mathbf{A}
$$$$
\text { 5: } \mathbf{V}=\mathbf{Q U}
$$

6: $\mathbf{Z}=\mathbf{V} \boldsymbol{\Lambda} \mathbf{V}^{T}$

\section{$\triangleright$ Sampling $\mathbf{Z}$ with $\mathbf{X}$}

$\triangleright$ QR decomposition on $\mathbf{Y}$

$\triangleright$ Solve for $\mathbf{A}$

$\triangleright$ SVD on A

In our application, we have $\mathbf{Z}=\mathbf{B}_{n+1}-\mathbf{I}$. The key to combining SRVM and randomised SVD is how to construct $\mathbf{Y}$ from algorithm (2). For each random vector $\mathbf{x}$ within $\mathbf{X}$, we have a sampled vector $\mathbf{y}$ for $\mathbf{Y}$ as 


\section{Algorithm 4 Sampling vector $\mathrm{y}$ for $\mathbf{Y}$}

121

$$
\begin{aligned}
& \text { 1: } \mathbf{d}=-\mathbf{S}_{n+1}^{T} \mathbf{x}=\prod_{i=0}^{n}\left(\frac{v_{i}}{P_{i}} \mathbf{w}_{i} \mathbf{w}_{i}^{T}-\mathbf{I}\right) \mathbf{x}=\prod_{i=0}^{n}\left[\frac{v_{i}}{P_{i}}\left(\mathbf{w}_{i}^{T} \mathbf{x}\right) \mathbf{w}_{i}-\mathbf{x}\right] \\
& 2: \mathbf{y}=\mathbf{S}_{n+1} \mathbf{S}_{n+1}^{T} \mathbf{x}=\mathbf{S}_{n+1} \mathbf{d}=\prod_{i=0}^{n}\left(\mathbf{I}-\frac{v_{i}}{P_{i}} \mathbf{w}_{i} \mathbf{w}_{i}^{T}\right) \mathbf{d}=\prod_{i=0}^{n}\left[\mathbf{d}-\frac{v_{i}}{P_{i}}\left(\mathbf{w}_{i}^{T} \mathbf{d}\right) \mathbf{w}_{i}\right]
\end{aligned}
$$

3: $\mathbf{y}=\mathbf{y}-\mathbf{x}$

We run such kind of random samplings on $\mathbf{Z}$ with a collection of independent $\mathbf{x}$ for $n_{\text {iter }}$ times, with $n_{\text {iter }}$ being the iteration number at convergence, because $\mathbf{Z}$ has already been in a low-rank form in algorithm (1). Then, we merge the resulting $\mathbf{y}$ collection into a matrix $\mathbf{Y}$ to continue the workflow in algorithm (3), whose verification can be found in Liu and Peter (2019). Finally, we can represent the inverse Hessian in a SVD form as

$$
\mathbf{H}^{-1}=\mathbf{V} \boldsymbol{\Lambda} \mathbf{V}^{T}
$$

123

in which the eigenvectors of $\mathbf{H}^{-1}$ are ordered within $\mathbf{V}$ and the eigenvalues within $\boldsymbol{\Lambda}$. In the next subsection, we will introduce the SRVM-based nullspace shuttle from the subspace spanned from $\mathbf{V}$.

\subsection{SRVM-based nullspace shuttle}

In this part, we attempt to construct the SRVM-based nullspace shuttle. Deal and Nolet (1996) project the model-space changes onto the nullspace of the data-misfit Hessian $\mathbf{G}^{T} \mathbf{G}$. However, the estimation of the full nullspace eigenvectors is infeasible, because it would require many more eigenvectors than that of the effective-space eigenvectors (Deal \& Nolet, 1996). Also, it is hard to define the noiseless nullspace eigenvectors. Finally, Deal and Nolet (1996) define the projection matrix for the nullspace shuttle by subtracting the resolution matrix constructed by the effective-space eigenvectors from the ideal resolution matrix, i.e., the identity matrix. Suppose we could succeed in finding the full, noiseless nullspace eigenvectors in a nonsingular $\mathbf{G}^{T} \mathbf{G}$, then this would correspond to the subspace spanned by the last smallest eigenvalues. Subsequently, if we take the inverse of $\mathbf{G}^{T} \mathbf{G}$, these nullspace eigenvectors should correspond to the first largest eigenvectors of $\left(\mathbf{G}^{T} \mathbf{G}\right)^{-1}=\mathbf{H}^{-1}$. Based on this assumption, in this paper, we propose to take the effective nullspace eigenvector(s) of $\mathbf{G}^{T} \mathbf{G}$ as the first largest eigenvector of $\mathbf{V}$. The definition of the nullspace eigenvector is not 
enough, because we need to design a nullspace shuttle by constructing the conservatively filtered models a posteriori.

The uncertainty in model parameters can be measured by the resolution (Boschi, 2003) or covariance (Soldati \& Boschi, 2005) matrices. Here, we consider the construction of the conservative models from the posterior samplings (Tarantola, 2005) as

$$
\mathbf{m}_{\text {post }}=\widetilde{\mathbf{m}}+\mathbf{C}_{\text {post }}^{1 / 2} \mathbf{n},
$$

with $\widetilde{\mathbf{m}}$ being the inverted model (also known as the maximum a posteriori model), $\mathbf{C}_{\text {post }}$ being the posterior covariance matrix, $\mathbf{n}$ of zero mean and banded covariance being a $2 \mathrm{D} / 3 \mathrm{D}$ Gaussian random sampler in the 2D/3D case (Tarantola, 2005). Strictly speaking, $\mathbf{n}$ should be a Gaussian random vector with zero mean and identity covariance. We put the banded effect into the 2D/3D Gaussian random fields to enable a diagonal representation of the prior covariance matrix for large-scale applications. The posterior covariance of a linear inverse problem with Gaussian priors is defined as

$$
\mathbf{C}_{\text {post }}=\left(\mathbf{G}^{T} \mathbf{C}_{d}^{-1} \mathbf{G}+\varepsilon \mathbf{C}_{\text {prior }}^{-1}\right)^{-1}
$$

with $\mathbf{C}_{d}$ being the data covariance matrix, $\mathbf{C}_{\text {prior }}$ and $\mathbf{C}_{\text {post }}$ being the prior and posterior model covariance matrices, respectively, and $\varepsilon$ being a tuning scalar upon $\mathbf{C}_{\text {prior }}$ for stabilization. The a priori knowledge of $\mathbf{C}_{d}$ and $\mathbf{C}_{\text {prior }}$ can prove helpful in seismic inversions ${ }_{147}$ (de Wit et al., 2012). Herein, we focus on the role of the SRVM-based inverse Hessian in the determination of the nullspace shuttle, leaving the precise estimation regarding the data covariance and prior model covariance matrices an open question for future research.

We can rewrite equation (11) as

$$
\mathbf{C}_{\text {post }}=\left(\mathbf{G}^{T} \mathbf{C}_{d}^{-1} \mathbf{G}+\varepsilon \mathbf{C}_{\text {prior }}^{-1}\right)^{-1}=\left(\mathbf{C}_{\text {prior }}^{1 / 2} \mathbf{G}^{T} \mathbf{C}_{d}^{-1} \mathbf{G C}_{\text {prior }}^{1 / 2}+\varepsilon \mathbf{I}\right)^{-1} \mathbf{C}_{\text {prior }}
$$

Absorbing $\mathbf{C}_{\text {prior }}^{1 / 2}$ into $\mathbf{G} \mathbf{C}_{\text {prior }}^{1 / 2}$ and $\mathbf{C}_{\text {prior }}^{1 / 2} \mathbf{G}^{T}$ means that the prior covariance matrix takes part in the linearized inversion of elastic FWI, leading to an approximate inverse data-misfit Hessian as $\mathbf{H}^{-1} \approx\left(\mathbf{C}_{\text {prior }}^{1 / 2} \mathbf{G}^{T} \mathbf{C}_{d}^{-1} \mathbf{G} \mathbf{C}_{\text {prior }}^{1 / 2}+\varepsilon \mathbf{I}\right)^{-1}$. Then, we have

$$
\mathbf{C}_{\text {post }} \approx \mathbf{H}^{-1} \mathbf{C}_{\text {prior }}
$$

in which the inverse data-misfit Hessian $\mathbf{H}^{-1}$ connects the prior model covariance $\mathbf{C}_{\text {prior }}$ to the posterior model covariance $\mathbf{C}_{\text {post }}$. We can thus rewrite equation () as

$$
\mathbf{m}_{\text {post }}=\widetilde{\mathbf{m}}+\mathbf{C}_{\text {prior }}^{1 / 2}\left[\mathbf{V} \boldsymbol{\Lambda}^{1 / 2} \mathbf{V}^{T}\right] \mathbf{n} .
$$


By taking the first largest eigenvalue $\lambda_{1}$ from $\boldsymbol{\Lambda}$ and the corresponding eigenvector $\mathbf{v}_{1}$ from V, we construct the SRVM-based conservatively filtered models a posteriori as

$$
\mathbf{m}_{c}=\widetilde{\mathbf{m}}+\mathbf{C}_{\text {prior }}^{1 / 2}\left[\mathbf{v}_{1} \lambda_{1}^{1 / 2} \mathbf{v}_{1}^{T}\right] \mathbf{n}
$$
root diagonals of $\mathbf{C}_{\text {post }} \approx \mathbf{H}^{-1} \mathbf{C}_{\text {prior }}=\left[\mathbf{V} \boldsymbol{\Lambda} \mathbf{V}^{T}\right] \mathbf{C}_{\text {prior }}$ provide a quantitative measure of the posterior uncertainty. Among $\mathbf{V}$ and $\boldsymbol{\Lambda}$, the first eigenvector $\mathbf{v}_{1}$ together with the first eigenvalue $\lambda_{1}$ contributes the most to the $\mathbf{C}_{\text {post }}$ based uncertainty estimation. It also means that the inverted structures associated with $\mathbf{C}_{\text {prior }}^{1 / 2}\left[\mathbf{v}_{1} \lambda_{1}^{1 / 2} \mathbf{v}_{1}^{T}\right]$ are with the least data coverage from the data-misfit Hessian point of view. If we look further into the term $\mathbf{C}_{\text {prior }}^{1 / 2}\left[\mathbf{v}_{1} \lambda_{1}^{1 / 2} \mathbf{v}_{1}^{T}\right] \mathbf{n}$, we will figure out that the product $\lambda_{1} \mathbf{v}_{1}^{T} \mathbf{n}$ is a scalar. We can, therefore, simplify equation (15) into

$$
\mathbf{m}_{c}=\widetilde{\mathbf{m}}+\mathbf{C}_{\text {prior }}^{1 / 2} \mathbf{v}_{1} \eta(\mathbf{n})
$$

in which $\eta(\mathbf{n})=\lambda_{1}^{1 / 2} \mathbf{v}_{1}^{T} \mathbf{n}$, a scalar function regarding $\mathbf{n}$, acts as a scaling factor to $\mathbf{C}_{\text {prior }}^{1 / 2} \mathbf{v}_{1}$, and $\mathbf{C}_{\text {prior }}^{1 / 2} \mathbf{v}_{1} \eta(\mathbf{n})$ becomes the nullspace shuttle $\delta \mathbf{m}_{\text {null }}$.

\section{Numerical example}

Elastic FWI mainly consists of three recursive steps: (i) gradient computation by the adjoint method, (ii) gradient update with the optimization method, and (iii) the steplength estimation with the line search. For the gradient computation, the solution to the 
elastic FWI depends on the model parameterization, and subsequently the sensitivity kernels. There are different parameterization strategies (Trampert \& Van Der Hilst, 2005). For our model parameters, we focus on the inversion of $V_{P}$ and $V_{S}$ velocities, but keep density fixed due to its weak sensitivity in waveform inversion (Virieux \& Operto, 2009). For the optimization algorithm in elastic FWI, we employ the SRVM method (Liu et al., 2019) and compare its result to an L-BFGS approach as a reference (Nocedal \& Wright, 2006). For the determination of the search step length, we employ the inexact line search together with the Wolfe conditions (Nocedal \& Wright, 2006).

\subsection{Elastic Marmousi Benchmark}

We take the elastic Marmousi (Martin, Wiley, \& Marfurt, 2006) benchmark for demonstration of our methods. The model is $9200 \mathrm{~m}$ long and $3000 \mathrm{~m}$ deep. Figures $1 \mathrm{a}$ and $1 \mathrm{~b}$ show the true and initial models, respectively. For the initial model, we convolve the true model with a Gaussian smoother wide enough to blur imprints of the discontinuities. We extend the models with convolutional perfectly matched layers (CPMLs) (Komatitsch \& Martin, 2007). There are 32 shots and 500 receivers evenly distributed. The source function is a 4-Hz Ricker wavelet. We run the numerical simulations with SPECFEM2D (Komatitsch \& Vilotte, 1998), and manage the inversion workflow with Seisflows (Modrak \& Tromp, 2016).

The resulting (maximum a posteriori) models by L-BFGS and SRVM-based elastic FWIs are shown in Figures 1c and 1d, respectively. For a fair comparison, we carry forward the inversions until either of them meets the stopping criterion. The red curve in Figure 1e shows that the SRVM-based elastic FWI stops at the 58-th iteration, with the normalized data-misfit minimization at $1.31 \times 10^{-2}$. The blue curve in Figure 1e shows the convergence behavior of the L-BFGS based elastic FWI as a reference. SRVM performs as good as L-BFGS in this elastic FWI example. For more details about the performance of SRVM against L-BFGS approaches in elastic FWI, we refer to Liu et al. (2019). Using the vectorversion SRVM algorithm, after 58 iterations we have stored 58 SRVM scalars and vectors. We will use this set of scalars and vectors to reconstruct the data-misfit inverse Hessian for the SRVM-based nullspace shuttle. The matrix reconstruction with the scalars and vectors can be understood as a low-rank approximation of the inverse Hessian.

To facilitate the information retrieval from the SRVM-based inverse Hessian, we incorporate the single-channel randomised SVD (Halko et al., 2011) into the SRVM matrix 

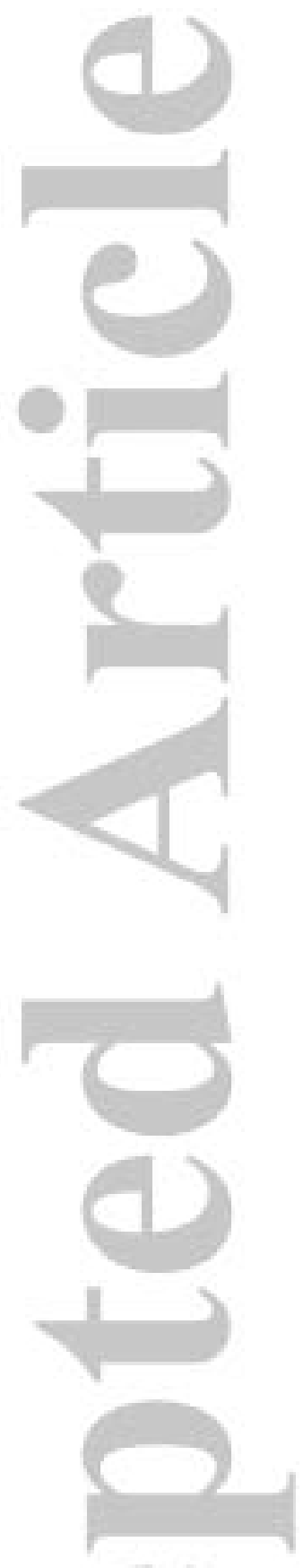

Figure 1: Recovery of the elastic Marmousi model for two different synthetic FWIs using SRVM and L-BFGS. Plotted are (a) the true elastic Marmousi model; (b) the initial model for elastic FWI; (c) and (d) the inverted models by L-BFGS and SRVM-based elastic FWIs, respectively; and (e) the data misfit convergence curves of L-BFGS and SRVM-based elastic FWIs (Left panel: $V_{P}$, Right panel: $V_{S}$ velocities, in $\mathrm{m} / \mathrm{s}$ ).

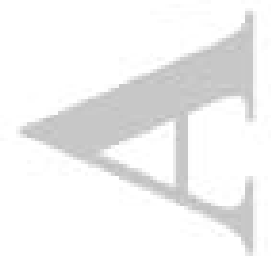


retrieval algorithm. Because the rank order of the factorized matrix by randomised SVD is the same with that of the original matrix, we choose the set of random samplers composed of 58 independent $1 \mathrm{D}$ random vectors. Note that $1 \mathrm{D}$ random vectors are qualified for the randomised SVD (Halko et al., 2011) and it avoids the more time-consuming generation of 2D/3D random samplers. Figure 2a shows the eigenvalues of the SRVM-based inverse Hessian. The rest of Figure 2 displays five different eigenvectors associated with different eigenvalues. An interesting observation is that the energy distributions of the eigenvectors gradually move from the bottom to top of the subsurface model as the eigenvector order increases. We can reason this tendency from the inverse of $\mathbf{H}^{-1}$, i.e., the Hessian, in which the energy distribution should fade away from top to bottom as the eigenvector order increases (Bui-Thanh et al., 2013; Zhu et al., 2016).

As discussed in equation (16), we explore to construct the nullspace shuttle via the first, primary eigenvector of $\mathbf{H}^{-1}$. We start with the investigation of the standard deviation maps from the square-root diagonals of the posterior covariance $\mathbf{C}_{\text {post }}$, which involves the prior covariance matrix $\mathbf{C}_{\text {prior }}$. In this research, we leave the estimation about $\mathbf{C}_{\text {prior }}$ as an open question and set the same standard deviation of $250 \mathrm{~m} / \mathrm{s}$ everywhere. Thus, the prior model perturbation distributions range between $[-1000 \mathrm{~m} / \mathrm{s}, 1000 \mathrm{~m} / \mathrm{s}]$ under a 2D Gaussian distribution (Tarantola, 2005). Figure 3a shows a 2D standard deviation map from the posterior covariance, and figure $3 \mathrm{~b}$ the contribution of the first eigenvector in Figure $2 \mathrm{a}$ to the map. The standard deviation maps indicate the uncertainties we have on the maximum a posteriori model. The $V_{P}$ map has more substantial standard deviations than $V_{S}$ because the $V_{S}$ sensitivity kernel possesses more low-wavenumber components than the $V_{P}$ kernel (Tarantola, 1986; Virieux \& Operto, 2009; Wu \& Aki, 1985). We can see that the first eigenvector dominates the uncertainties in Figure 3. Note that in some very marginal areas (e.g., the PMLs), their uncertainties remain zero. This occurs because we cannot adjust areas where the Fréchet sensitivity kernels remain zero. Similar explanations can be found in Kennett, Sambridge, and Williamson (1988) and Rawlinson, Fichtner, Sambridge, and Young (2014). Based on these facts, we will take the first eigenvector of the inverse data-misfit Hessian to evaluate the robustness and extract non-unique solutions from a tomographic image.

From equation (16) we see that it is possible to investigate the posterior model distributions with 2D Gaussian random fields, as shown in Figure 4a. Also, equation (16) indicates that $\mathbf{v}_{1}^{T} \mathbf{n}$ is a scalar whose value is a function regarding $\mathbf{n}$. Figure 4 a shows two Gaussian 


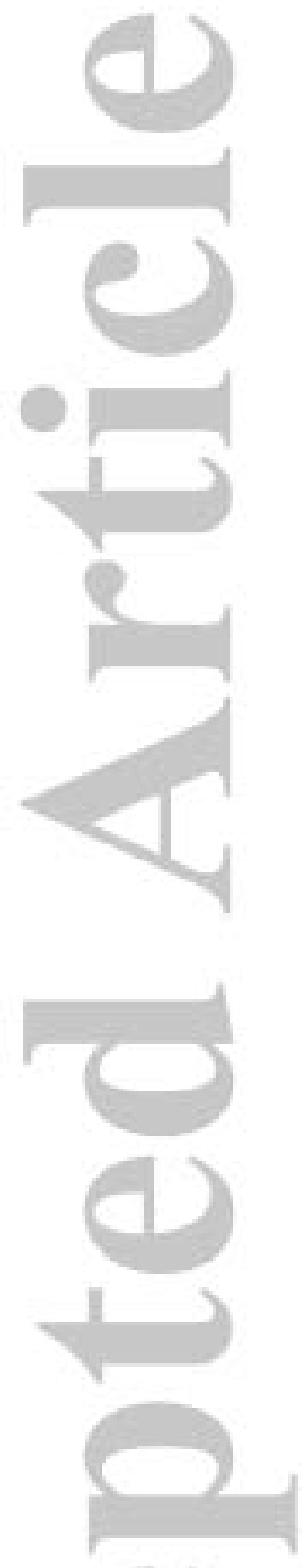

Figure 2: Eigen-decomposition of the SRVM-based inverse Hessian factorized by randomised SVD. Plotted in (a) is the eigenvalues $\lambda_{i}$ up to rank 58 on logarithmic scale. Plotted in the rest are the $1 \mathrm{st}, 5 \mathrm{th}, 10 \mathrm{th}, 20 \mathrm{th}$, and 58 th eigenvectors, respectively, $\mathbf{v}_{i}$ of the inverse Hessian (Left panel: $V_{P}$, Right panel: $V_{S}$ velocities, in $\mathrm{m} / \mathrm{s}$ ). Each eigenvector integrates to 1 over the spatial domain.

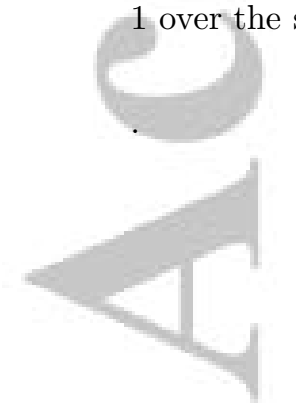




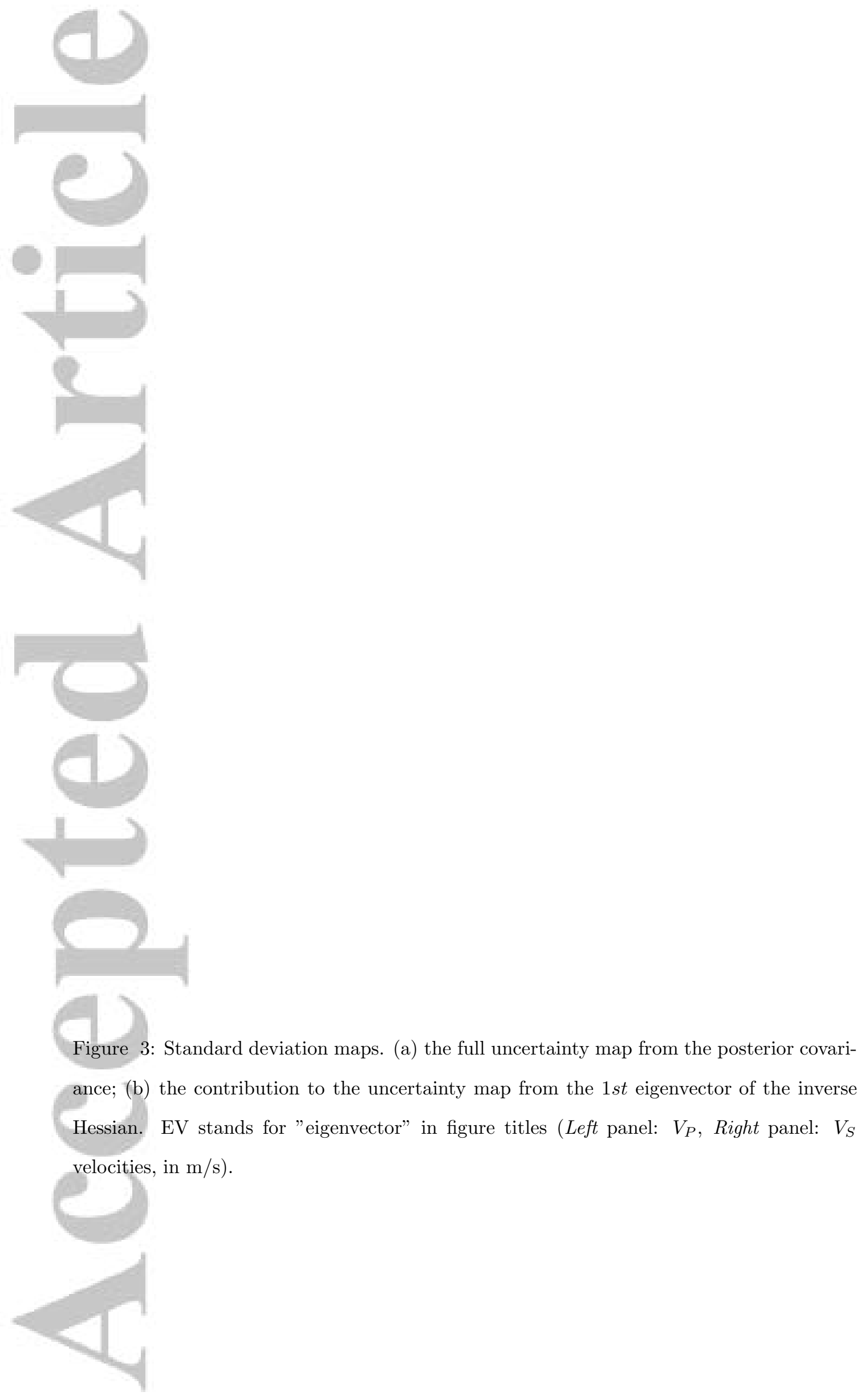


random fields (i.e., $\mathbf{n})$. Figure $4 \mathrm{~b}$ shows a nullspace shuttle generated by $\mathbf{C}_{\text {prior }}^{1 / 2} \mathbf{v}_{1} \eta(\mathbf{n})$ from the SRVM-based elastic FWI. Note that with different $\mathbf{n}$, we may generate thousands of different nullspace shuttles. Rather than a path, a shuttle here is a vehicle, which indicates one perturbed model in the null space spanned by $\mathbf{v}_{1}$. All the shuttles only differ from figures in the top row of Figure 2a in magnitude. Note that in Figure 2, we use a normalized colorbar for all the subfigures, but here in Figure $4 \mathrm{~b}$, we set the limits of the colorbar to $90 \%$ of its respective absolute maximum. The absolute maximum values for the posterior $V_{P}$ and $V_{S}$ perturbations in Figure $4 \mathrm{~b}$ are around 150 and 114, respectively. Also, in Figure 4b, we shrink the grey-color scope, which connects the blue and red zones on the colorbar, for a better visualization regarding the structures of different magnitudes.

We use equation (16) to construct different conservatively filtered models. Again, the inverted (maximum a posteriori) model is shown as reference in Figure 5a, with the normalized optimal data misfit value given in the figure title. The other subfigures in Figure 5 show, respectively, the inverted models $\widetilde{\mathbf{m}}$ plus $\mathbf{C}_{\text {prior }}^{1 / 2} \mathbf{v}_{1} \eta(\mathbf{n})$ amplified by $-4,-2,+2$, +4 , with the corresponding data misfits of the conservative models being displayed on their titles. Each of the data misfits is computed by actually running a forward simulation. With the maximum perturbation in Figure 4b being $150 \mathrm{~m} / \mathrm{s}$, from the conservative models we can see that although the structures within the model nullspace perturb between [-600 m/s, 600 $\mathrm{m} / \mathrm{s}$, only slight data-misfit distortions arise. Within a conservative choice of data-misfit tolerance (i.e., $1 \%$ of the total data misfit), we can find a broad range of acceptable models, indicating the non-unique solutions in elastic FWI. Because the $\mathbf{C}_{\text {prior }}^{1 / 2} \mathbf{v}_{1} \eta(\mathbf{n})$ is implemented a posteriori, we can generate thousands of conservative models in a highly efficient manner. That is also the reason for it being named the "nullspace shuttle". Realizations of the nullspace shuttle show that shallower subsurface structures with better data coverage are robust, the deeper, marginal structures, especially for the high-velocity salt structures, are less robust due to the lack of data coverage. Such observations may help to mitigate the non-uniqueness or uncertainties regarding the tomographic images in future acquisitions.

\section{Discussions and Conclusion}

Investigating the nullspace shuttle in FWI applications is tantamount to studying the non-uniqueness of the associated inverse problem. Focusing on FWI, The nullspace shuttle allows for a significant change in the tomographic image but only with a slight data-misfit distortion. We construct an SRVM-based nullspace shuttle in elastic FWI, focusing on $V_{P}$ 


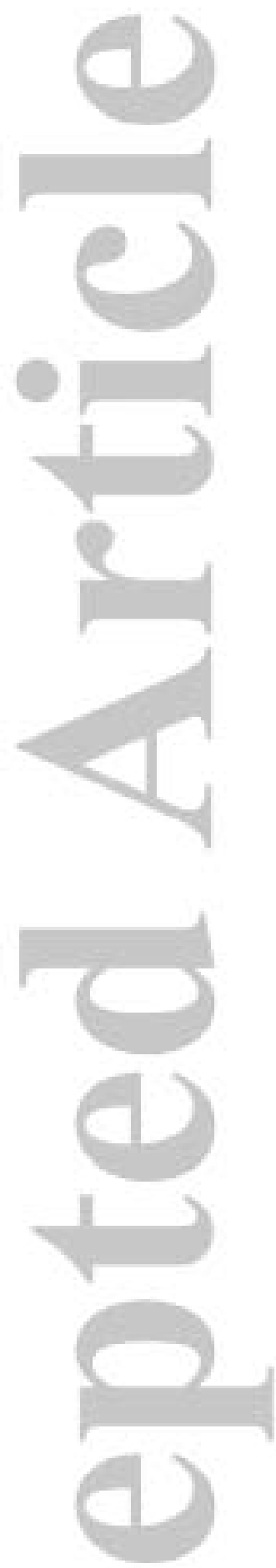

Figure 4: Nullspace shuttle. (a) Two 2D Gaussian random fields; (b) the sampled nullspace model perturbations (Left panel: $V_{P}$, Right panel: $V_{S}$ velocities, in m/s).

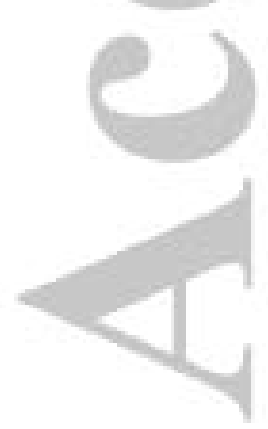




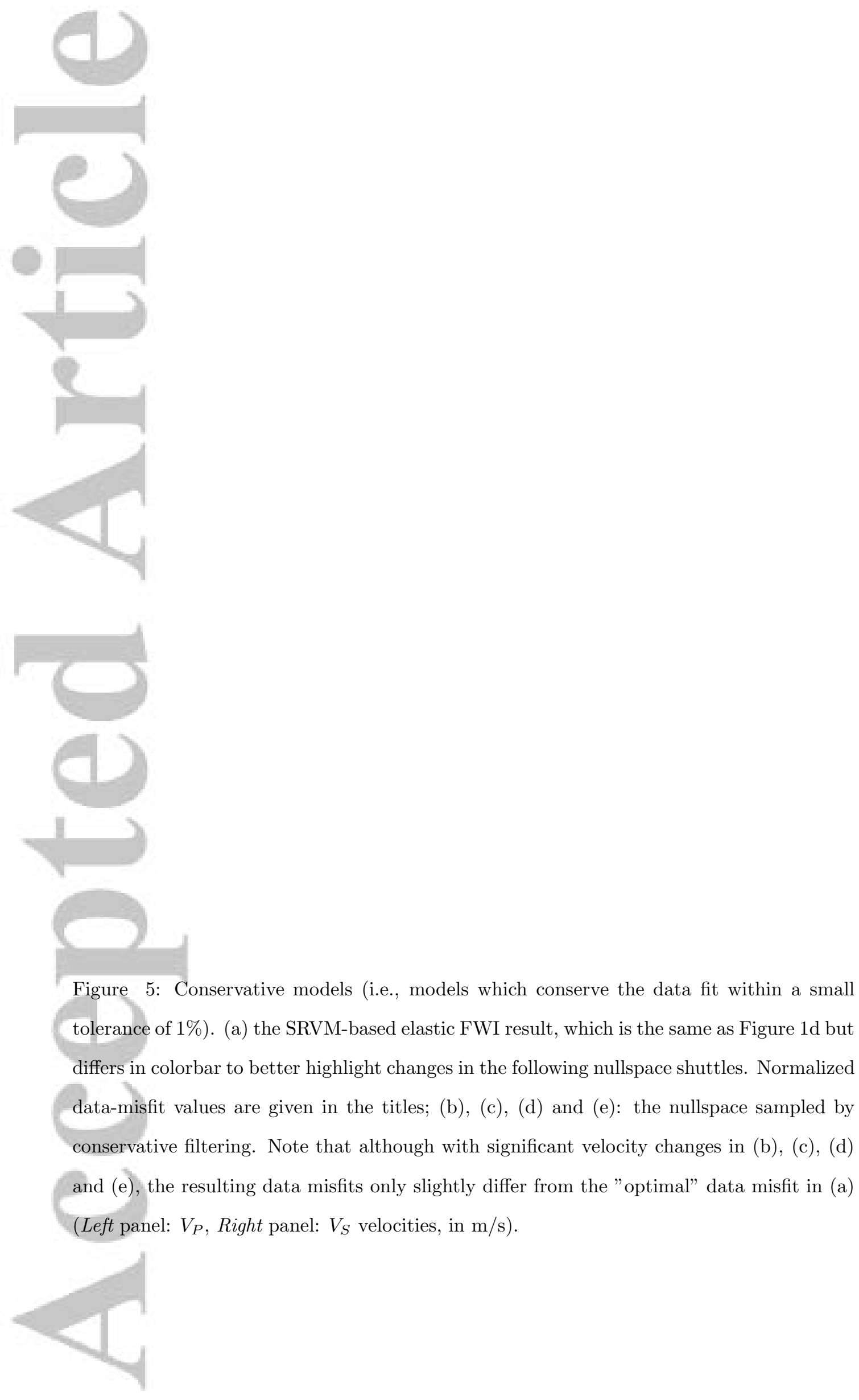


290

and $V_{S}$ velocity model inversions. To this end, we employ a vector-version SRVM algorithm which is memory-efficient and amends to large-scale tomographic problems. Hessian, we define the first, primary eigenvector of the SRVM-based inverse Hessian through randomised SVD as the generalized nullspace. We put this eigenvector into the posterior model sampling to construct the SRVM-based nullspace shuttle and conservative modelsampling. With the display of the nullspace shuttle, we observe that specific structures in the inverted models are not well resolved by the data assimilation. This means that these subsurface structures are less robust or unique, and should be interpreted with care. For better interpretation of these structures, changes in acquisition geometries or additional informations from well-logs or geological surveys would be recommended.

Because the SRVM method is deeply embedded in the elastic FWI procedure, the demonstrated application can be easily extended to other multi-parameter problems. The construction of the SRVM-based nullspace shuttle is straightforward once the approximated inverse Hessian is constructed. Essentially, our method resembles the Deal and Nolet (1996) nullspace shuttle but illuminates the problem from a complementary direction. Our numerical application highlights that this shuttle as well represents a characterization of the non-uniqueness in elastic FWI.

\section{Acknowledgments}

All data analyzed here are openly available. The Marmousi_SRVM model can be found at https://zenodo.org/badge/latestdoi/125932696. The Seisflow_SRVM codes can be found at https://zenodo.org/badge/latestdoi/125918416. The codes for null-space shuttle can be found at https://zenodo.org/badge/latestdoi/126001419. This work was supported by the King Abdullah University of Science \& Technology (KAUST) Office of Sponsored Research (OSR) under award No. UAPN\#2605-CRG4. Computational resources were provided by the Information Technology Division and Extreme Computing Research Center (ECRC) at KAUST.

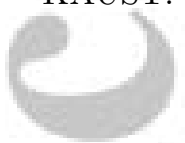

References

Backus, G. E., \& Gilbert, J. F. (1967). Numerical applications of a formalism for geophysical inverse problems. Geophys. J. R. astr. Soc., 13, 247-276. 
Boschi, L. (2003). Measures of resolution in global body wave tomography. Geophysical Rèsearch Letters, 30(19).

Bui-Thanh, T., Ghattas, O., Martin, J., \& Stadler, G. (2013). A computational framework for infinite-dimensional Bayesian inverse problems Part i: The linearized case, with application to global seismic inversion. SIAM Journal on Scientific Computing, 35(6), A2494-A2523.

Deal, M. M., \& Nolet, G. (1996). Nullspace shuttles. Geophysical Journal International, $124(2), 372-380$.

Deal, M. M., Nolet, G., \& van der Hilst, R. D. (1999). Slab temperature and thickness from seismic tomography: 1. method and application to tonga. Journal of Geophysical Research: Solid Earth, 104(B12), 28789-28802.

de Wit, R., Trampert, J., \& Hilst, R. (2012). Toward quantifying uncertainty in travel time tomography using the null-space shuttle. Journal of Geophysical Research: Solid Earth, 117(B3).

Fichtner, A., \& Leeuwen, T. v. (2015). Resolution analysis by random probing. Journal of Geophysical Research: Solid Earth, 120(8), 5549-5573.

Fichtner, A., \& Zunino, A. (2019). Hamiltonian nullspace shuttles. Geophysical Research Letters, 46, $644-651$.

Fichtner, A., Zunino, A., \& Gebraad, L. (2018). Hamiltonian monte carlo solution of tomographic inverse problems. Geophysical Journal International, 216(2), 1344-1363.

Fletcher, R., \& Powell, M. J. (1963). A rapidly convergent descent method for minimization. The computer journal, 6(2), 163-168.

Halko, N., Martinsson, P.-G., \& Tropp, J. A. (2011). Finding structure with randomness: Probabilistic algorithms for constructing approximate matrix decompositions. SIAM review, 53(2), 217-288.

Hull, D. G., \& Tapley, B. D. (1977). Square-root variable-metric methods for minimization. Journal of optimization theory and applications, 21(3), 251-259.

Kennett, B., Sambridge, M., \& Williamson, P. (1988). Subspace methods for large inverse problems with multiple parameter classes. Geophysical Journal International, 94(2), $237-247$.

Komatitsch, D., \& Martin, R. (2007). An unsplit convolutional Perfectly Matched Layer improved at grazing incidence for the seismic wave equation. Geophysics, 72(5), SM155SM167. doi: 10.1190/1.2757586 
Komatitsch, D., \& Vilotte, J. P. (1998). The spectral-element method: an efficient tool to simulate the seismic response of 2D and 3D geological structures. Bull. seism. Soc. Am., 88(2), 368-392.

Leweke, S., Michel, V., \& Telschow, R. (2018). On the non-uniqueness of gravitational and magnetic field data inversion (survey article). In W. Freeden \& M. Z. Nashed (Eds.), Handbook of mathematical geodesy: Functional analytic and potential theoretic methods (pp. 883-919). Cham: Springer International Publishing. Retrieved from https://doi.org/10.1007/978-3-319-57181-2_15 doi: 10.1007/978-3-319-57181-2 $-15$

Liberty, E., Woolfe, F., Martinsson, P.-G., Rokhlin, V., \& Tygert, M. (2007). Randomized algorithms for the low-rank approximation of matrices. Proceedings of the National Academy of Sciences, 104(51), 20167-20172.

Liu, Q. (2019). Acoustic full-waveform inversion and its uncertainty estimation based on a vector-version square-root variable metric method. Inverse Problems, 35(9), 095006.

Liu, Q., \& Peter, D. (2019). Square-root variable metric based elastic full-waveform inversionpart 2: uncertainty estimation. Geophysical Journal International, 218(2), $1100-1120$.

Liu, Q., Peter, D., \& Tape, C. (2019). Square-root variable metric based elastic fullwaveform inversion-part 1: theory and validation. Geophysical Journal International, $218(2), 1121-1135$.

Luo, Y. (2012). Seismic imaging and inversion based on spectral-element and adjoint methods. Ph.D. thesis.

Mai, P. M., Schorlemmer, D., Page, M., Ampuero, J.-P., Asano, K., Causse, M., ... others (2016). The earthquake-source inversion validation (siv) project. Seismological Research Letters, 87(3), 690-708.

Martin, G. S., Wiley, R., \& Marfurt, K. J. (2006). Marmousi2: An elastic upgrade for marmousi. The Leading Edge, 25(2), 156-166.

Meju, M. A. (2009). Regularized extremal bounds analysis (reba): An approach to quantifying uncertainty in nonlinear geophysical inverse problems. Geophysical Research Letters, 36(3).

Michel, V., \& Fokas, A. S. (2008). A unified approach to various techniques for the nonuniqueness of the inverse gravimetric problem and wavelet-based methods. Inverse problems, 24(045019), 25pp. 
Modrak, R., \& Tromp, J. (2016). Seismic waveform inversion best practices: regional, global and exploration test cases. Geophysical Journal International, 206(3), 1864-1889.

Morf, M., \& Kailath, T. (1975). Square-root algorithms for least-squares estimation. IEEE Transactions on Automatic Control, 20(4), 487-497.

Nocedal, J., \& Wright, S. (2006). Numerical optimization (2nd ed.). Springer-Verlag.

Nolet, G., \& Snieder, R. (1990). Solving large linear inverse problems by projection. Geophysical Journal International, 103(2), 565-568.

Paige, C. C., \& Saunders, M. A. (1982). Lsqr: An algorithm for sparse linear equations and sparse least squares. ACM Transactions on Mathematical Software (TOMS), 8(1), $43-71$.

Parker, R. (1977). Understanding inverse theory. Ann. Rev. Earth Planet. Sci., 5, 35-64.

Parker, R. (1994). Geophysical inverse theory. Princeton, NJ: Princeton University Press.

Pratt, R. G. (1999). Seismic waveform inversion in the frequency domain, part 1: Theory and verification in a physical scale model. Geophysics, 64(3), 888-901.

Rawlinson, N., Fichtner, A., Sambridge, M., \& Young, M. K. (2014). Chapter one-seismic tomography and the assessment of uncertainty. Advances in Geophysics, 55, 1-76.

Rowbotham, P. S., \& Pratt, R. G. (1997). Improved inversion through use of the null space. Geophysics, 62(3), 869-883.

Scales, J. A. (1987). Tomographic inversion via the conjugate gradient method. Geophysics, 52(2), 179-185.

Snieder, R., \& Trampert, J. (1999). Inverse problems in geophysics. In A. Wirgin (Ed.), Wavefield inversion (p. 119-190). New York: SpringerVerlag.

Soldati, G., \& Boschi, L. (2005). The resolution of whole earth seismic tomographic models. Geophysical Journal International, 161(1), 143-153.

Tarantola, A. (1986). A strategy for nonlinear elastic inversion of seismic reflection data. Geophysics, 51(10), 1893-1903.

Tarantola, A. (2005). Inverse problem theory and methods for model parameter estimation. Philadelphia, Pennsylvania, USA: Society for Industrial and Applied Mathematics.

Trampert, J., \& Spetzler, J. (2006). Surface wave tomography: finite-frequency effects lost in the null space. Geophysical Journal International, 164(2), 394-400.

Trampert, J., \& Van Der Hilst, R. D. (2005). Towards a quantitative interpretation of global seismic tomography. Geophysical Monograph Series, 160, 47-62.

Vasco, D. W., Lane, R., \& Majer, E. L. (1993). Ensemble inference in geophysical inverse 
problems. Geophys. J. Int., 115, 711-728.

393 Virieux, A., \& Operto, S. (2009). An overview of full-waveform inversion in exploration 394 geophysics. Geophysics, $74(6)$, WCC1-WCC26.

Williamson, W. E. (1975). Square-root algorithms for function minimization. AIAA Journal, 13(1), 107-109.

397 Wu, R.-S., \& Aki, K. (1985). Scattering characteristics of elastic waves by an elastic heterogeneity. Geophysics, 50(4), 582-595.

Zhu, H., Li, S., Fomel, S., Stadler, G., \& Ghattas, O. (2016). A Bayesian approach to 400 estimate uncertainty for full-waveform inversion using a priori information from depth migration. Geophysics, 81(5), R307-R323.
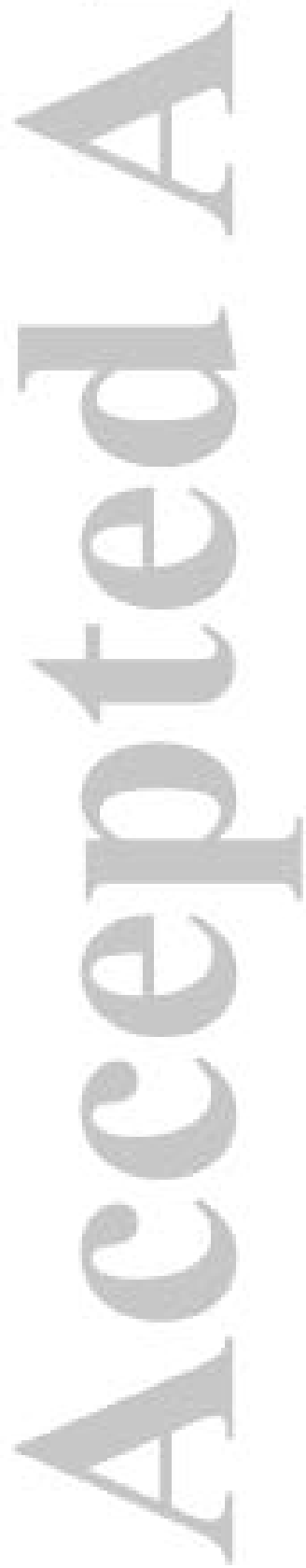
Figure 1.
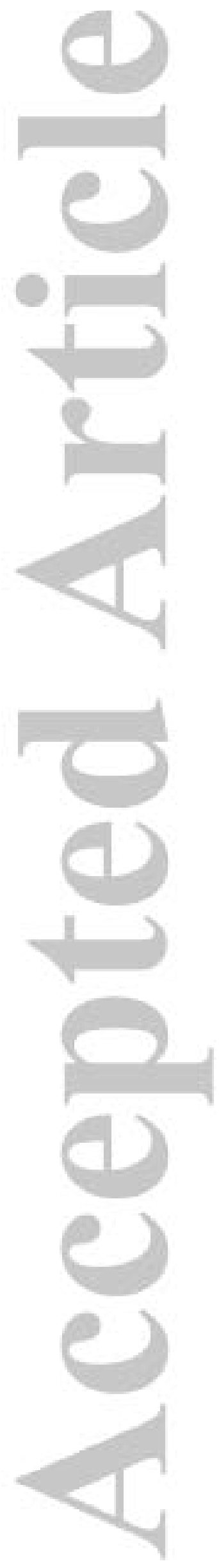

@2020 American Geophysical Union. All rights reserved. 


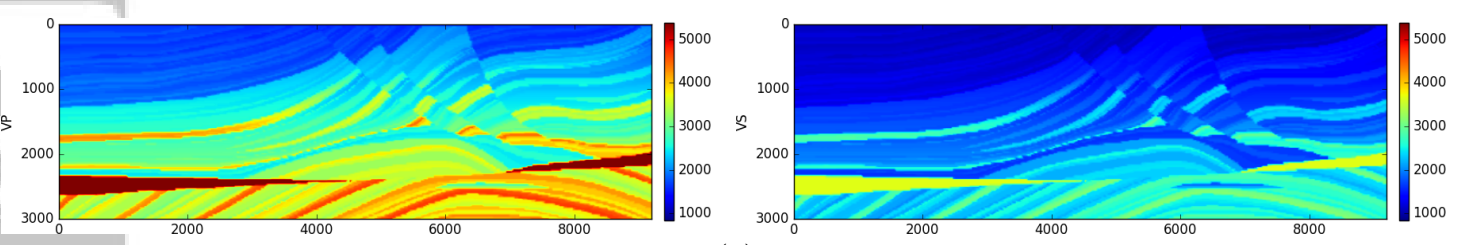

(a)
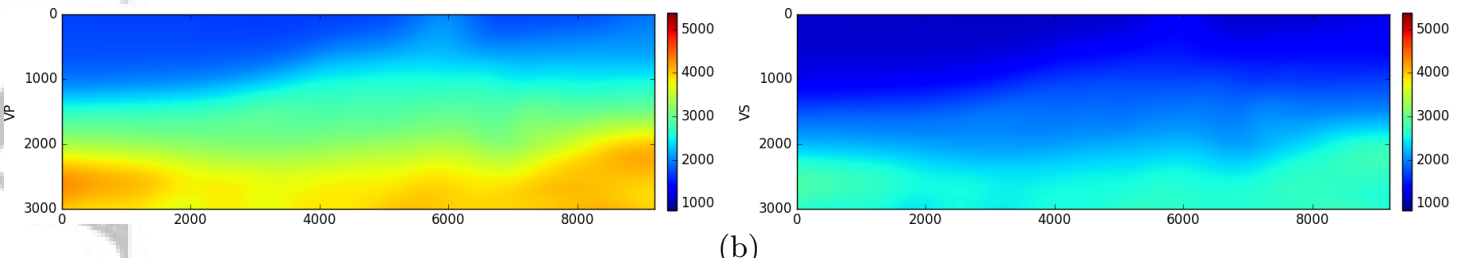

L-BFGS

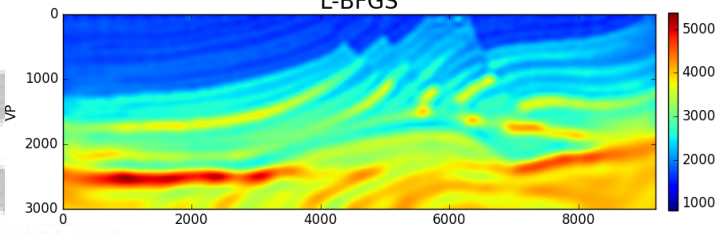

SRVM

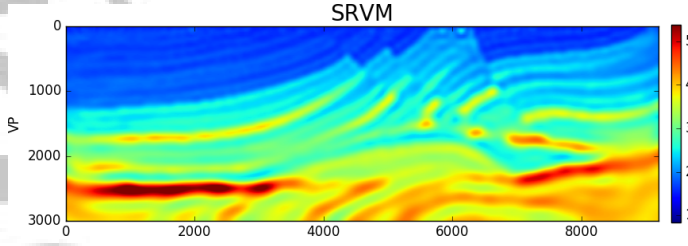

(d)

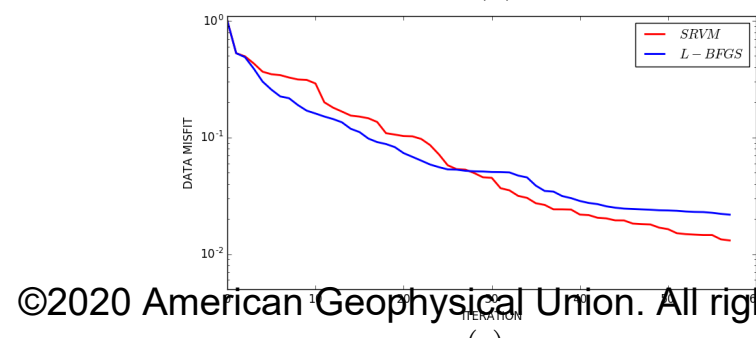

(C2020 American 'Geophysical Union. All rights reserved.

(e)

(c)

(b)
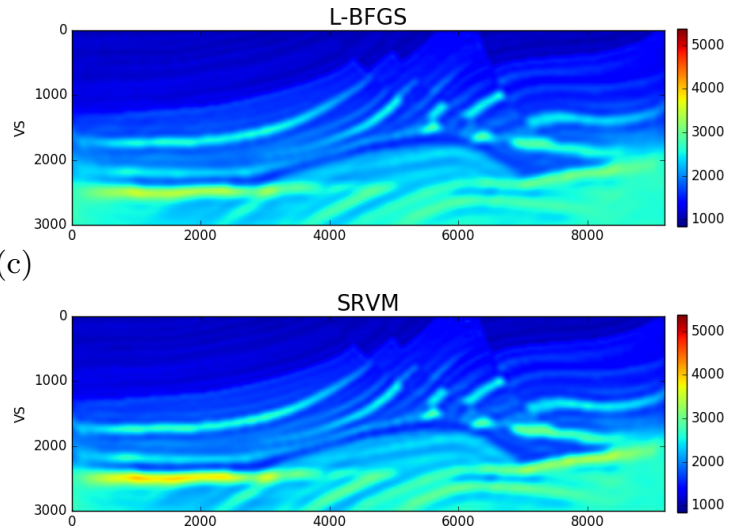
Figure 2.
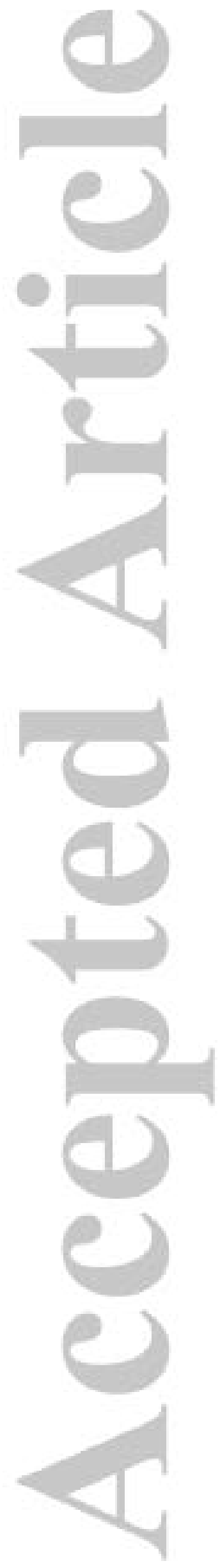

@2020 American Geophysical Union. All rights reserved. 


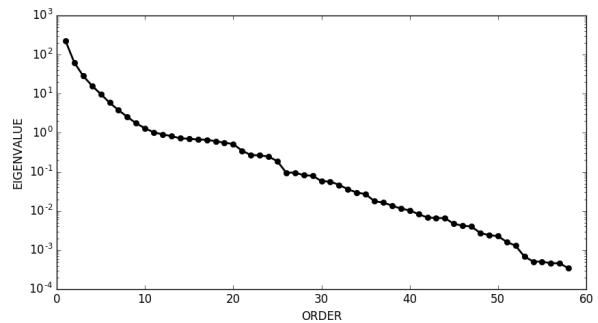

(a)
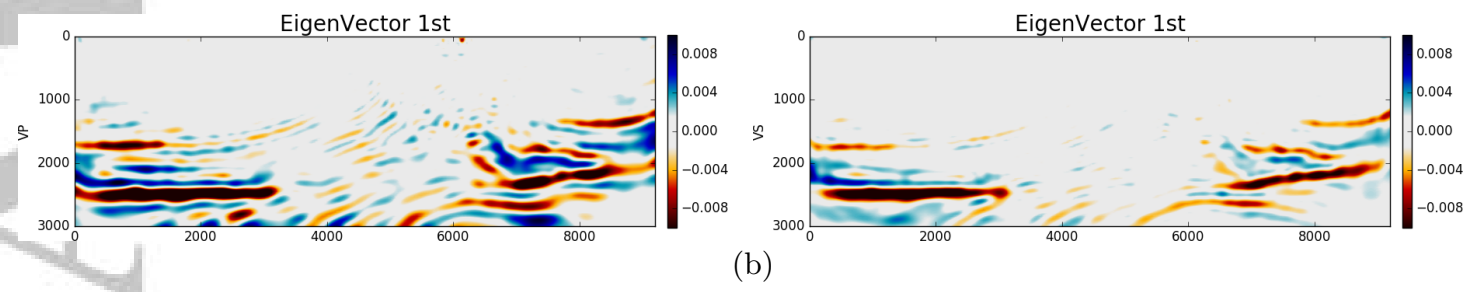

(b)
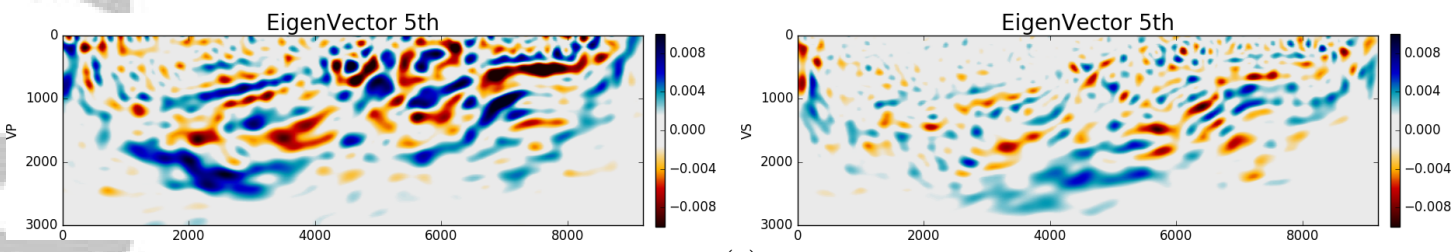

(c)

EigenVector 10th

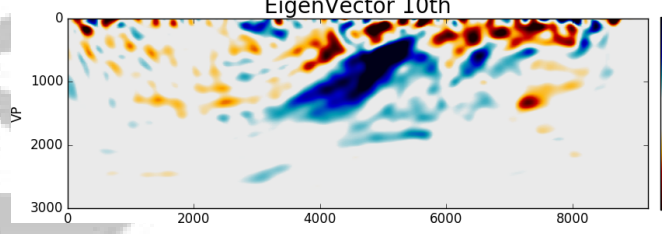

EigenVector 20th

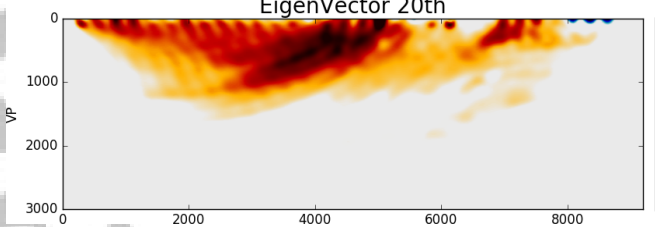

EigenVector 58th

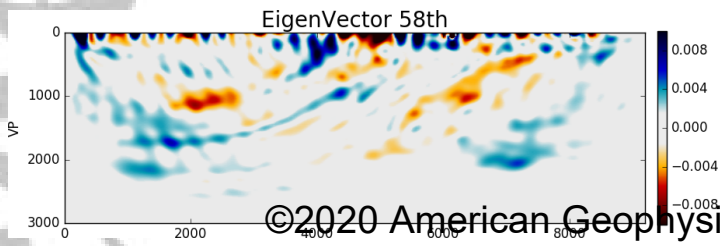

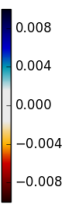

5

(d)

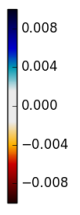

(e)

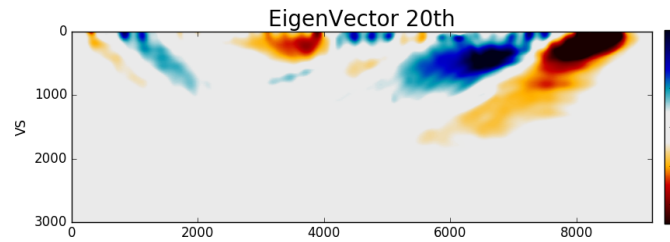

EigenVector 58th

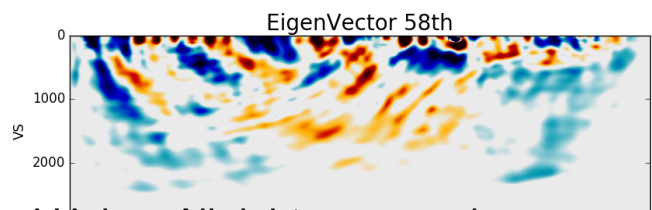

EigenVector 10th

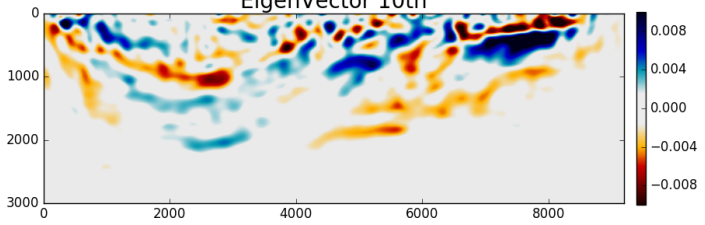

EigenVector 20th

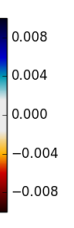

0.008
0.004
0.000
-0.004
-0.008

(f) 
Figure 3.
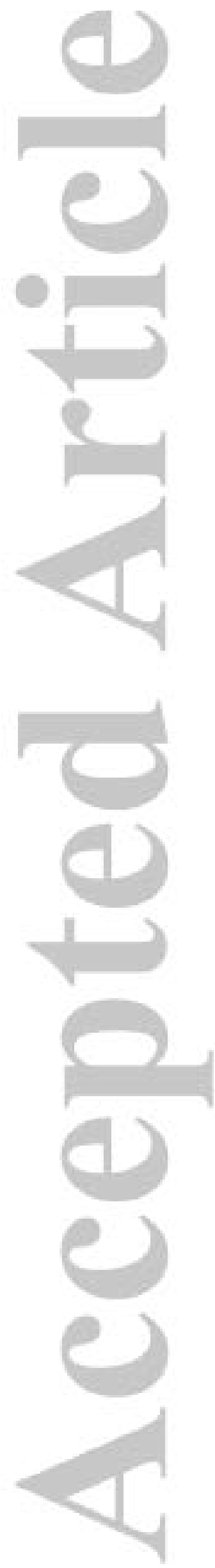

C2020 American Geophysical Union. All rights reserved. 
Figure 4.
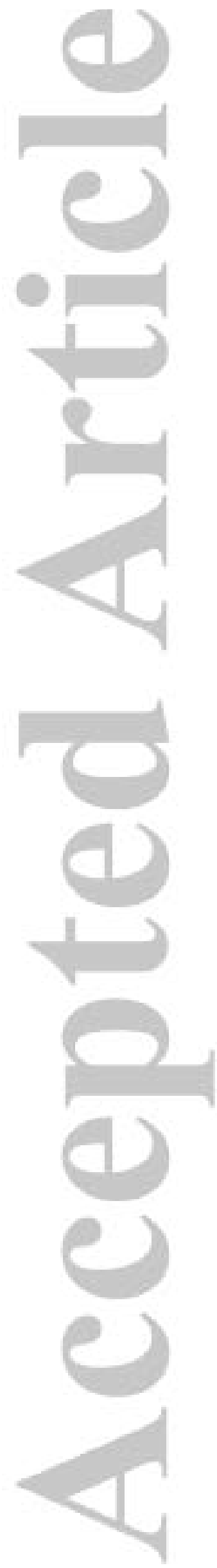

C2020 American Geophysical Union. All rights reserved. 
Figure 5.
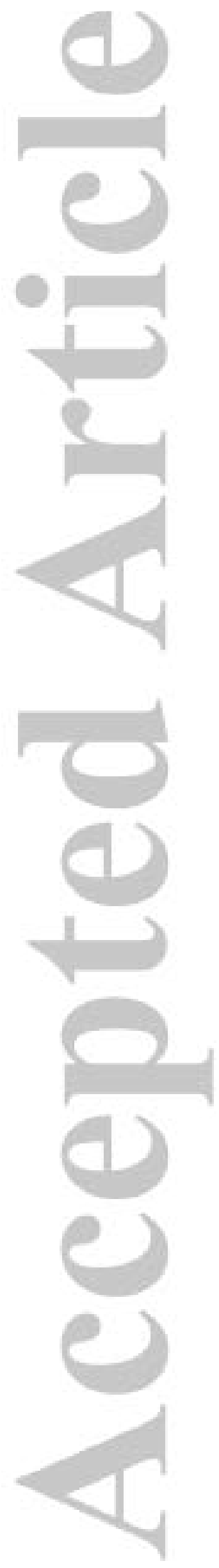

@2020 American Geophysical Union. All rights reserved. 
Inverted VP $\left(D_{\text {misfit }}=1.31 e-2\right)$
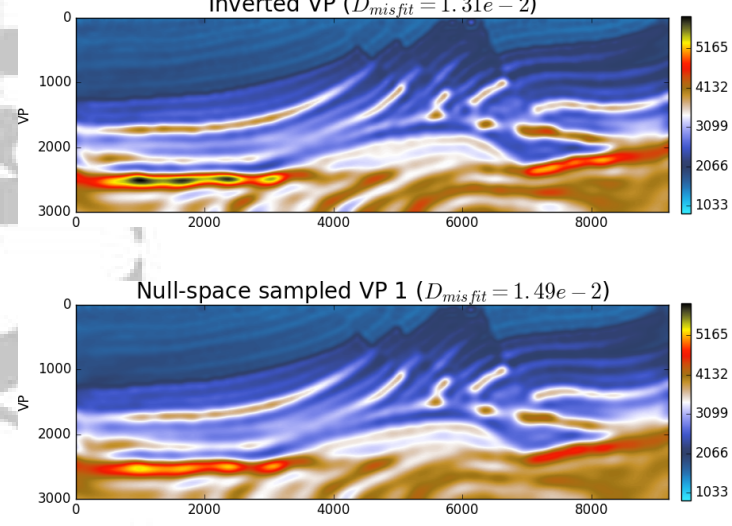

(a)

Inverted VS $\left(D_{\text {misfit }}=1.31 e-2\right)$
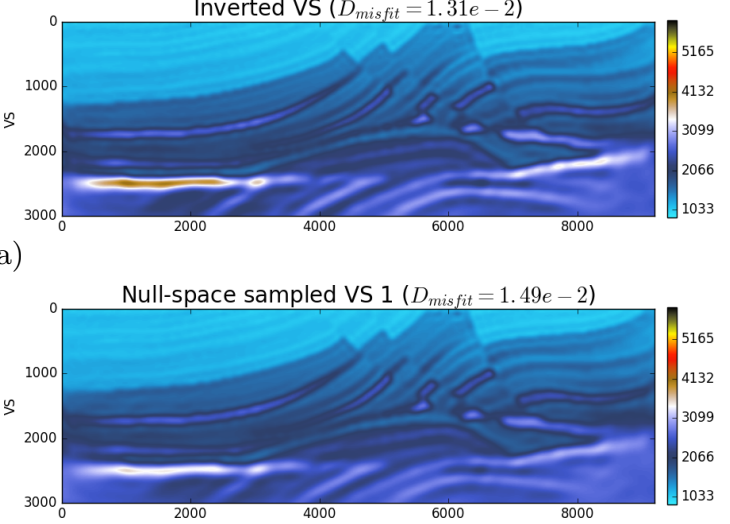

(b)

Null-space sampled VP $2\left(D_{\text {mis fit }}=1.32 e-2\right)$

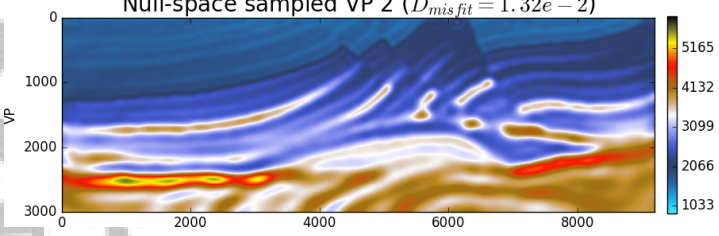

(c)

Null-space sampled VP $3\left(D_{\text {misfit }}=1.53 e-2\right)$

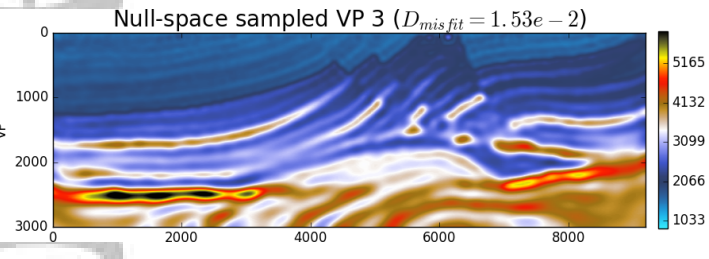

(d)

Null-space sampled VP $4\left(D_{\text {misfit }}=1.98 e-2\right)$
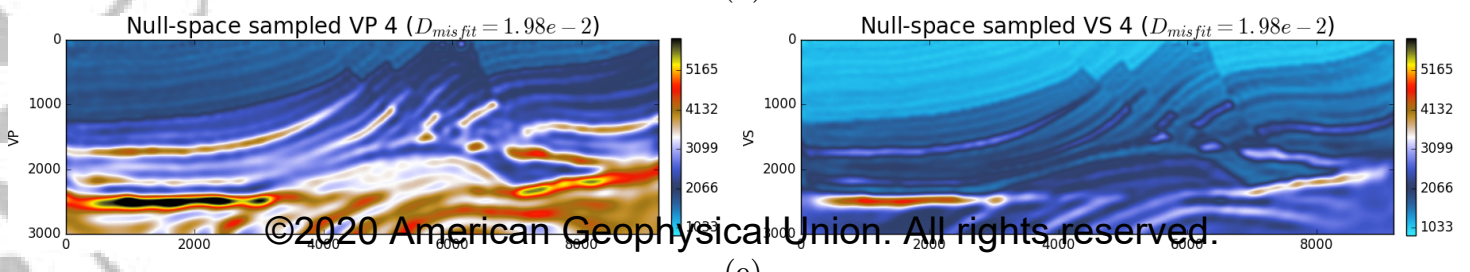

(e)
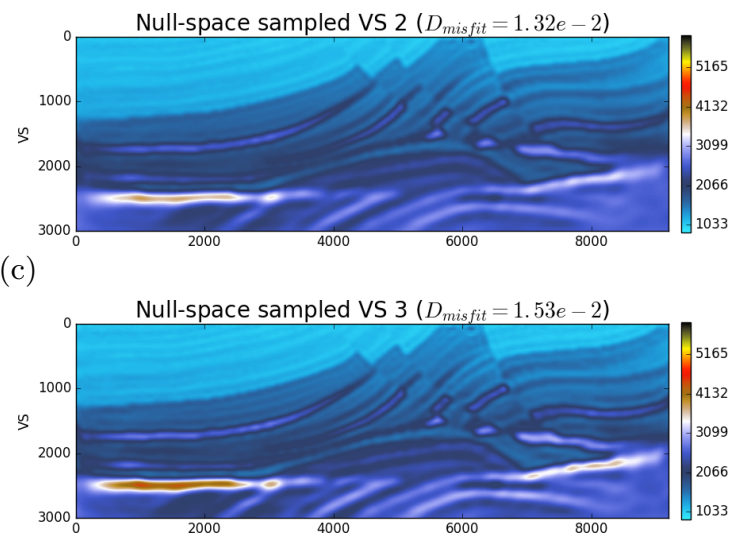